\title{
DYNAMIC CARDIOMYOPLASTY: ITS CHRONIC AND ACUTE EFFECTS ON THE FAILING HEART
}

Himanshu J. Patel, MD $^{\mathrm{a}}$

Edward B. Lankford, MD, PhD

David J. Polidori, MD $^{\mathrm{a}}$

James J. Pilla, PhD ${ }^{\mathrm{a}}$

Theodore Plappert, CVT $^{\mathrm{b}}$

Martin St. John Sutton, FRCP ${ }^{b}$

Michael A. Acker, MD $^{a}$

\begin{abstract}
Objectives: Dynamic cardiomyoplasty is an alternative therapy for end-stage heart failure. We investigated the mechanisms, both acute and chronic, by which a synchronously stimulated conditioned muscle wrap affects left ventricular function in a chronic canine model of dilated cardiomyopathy. Methods: Nineteen dogs underwent rapid ventricular pacing at a rate of 215 beats/min for 4 weeks to create a model of heart failure. Eight dogs were then randomly selected to undergo cardiomyoplasty, and all dogs received 6 additional weeks of rapid ventricular pacing. The cardiomyoplasty group also received a graded muscle conditioning protocol of synchronized burst stimulation to transform the muscle wrap. All dogs were studied with pressure-volume analysis and echocardiography at baseline and after 4 and 10 weeks of rapid ventricular pacing. Data in the cardiomyoplasty group were analyzed with the stimulator off, with it augmenting every beat (1:1), and with it augmenting only every other beat (1:2). Results: Stimulator "off" data at 10 weeks of rapid pacing demonstrated chronic effects by enhanced ventricular function (end-systolic elastance $=1.80$ after myoplasty vs 1.17 for controls, $p=0.005$ ) and a stabilization of volumes and composite end-systolic and end-diastolic pressure-volume relations in the cardiomyoplasty group when compared with controls. Myoplasty stimulation increased apparent contractility (preload recruitable stroke work $=31.3$ for stimulator "off" vs $\mathbf{4 0 . 6}$ for stimulator $1: 2$ assisted beats $[p<0.05]$ and vs 45.4 for stimulator $1: 1[p<0.05])$. Conclusions: Benefits from dynamic cardiomyoplasty are by at least two mechanisms: (1) the girdling effects of a conditioned muscle wrap, which halts the chronic remodeling of heart failure, and (2) active systolic assistance, which augments the apparent contractility of the failing heart. (J Thorac Cardiovasc Surg 1997;114:169-78)
\end{abstract}

$D^{s}$ ynamic cardiomyoplasty is a surgical option devised for the treatment of patients with endstage heart failure. In this procedure, the latissimus dorsi muscle is mobilized and wrapped circumferentially around the heart and is then stimulated synchronously with cardiac systole so that it can assist the failing myocardium. ${ }^{1}$ Preliminary results from the Food and Drug Administration's phase II trial

From the Divisions of Cardiothoracic Surgery ${ }^{\mathrm{a}}$ and Cardiology, ${ }^{\mathrm{b}}$ Departments of Surgery and Medicine, University of Pennsylvania School of Medicine, Philadelphia, $\mathrm{Pa}$.

Received for publication Dec. 10, 1996; revisions requested Feb. 5, 1997; revisions received March 4, 1997; accepted for publication March 5, 1997.

Address for reprints: Michael A. Acker, MD, Division of Cardiothoracic Surgery, Silverstein 6, Hospital of the University of Pennsylvania, 34th and Spruce Sts., Philadelphia, PA 19104.

Copyright (C) 1997 by Mosby-Year Book, Inc.

$0022-5223 / 97 \$ 5.00+0 \quad \mathbf{1 2 / 1 / 8 1 5 8 8}$ on cardiomyoplasty have shown beneficial effects on patient symptoms and cardiac function. ${ }^{2}$ The mechanism of action of cardiomyoplasty is yet to be determined, and discordant results on its effects on hemodynamics and cardiac function have been reported. Some studies have shown minimal effects of dynamic assistance, ${ }^{3-5}$ whereas others have documented improvement in function with added $\mathrm{dy}$ namic compression. ${ }^{6-14}$ Recently, some authors have suggested that the main mechanism of action may be a girdling effect of the muscle wrap, with ventricular volumes being stabilized by the presence of the wrap. $3,15-17$

These conflicting results can be attributed to several reasons. Some studies have been performed with the use of unconditioned muscle wraps, and other experiments have been performed on normal hearts. Neither of these situations is clinically relevant, because significant phenotypic changes occur 


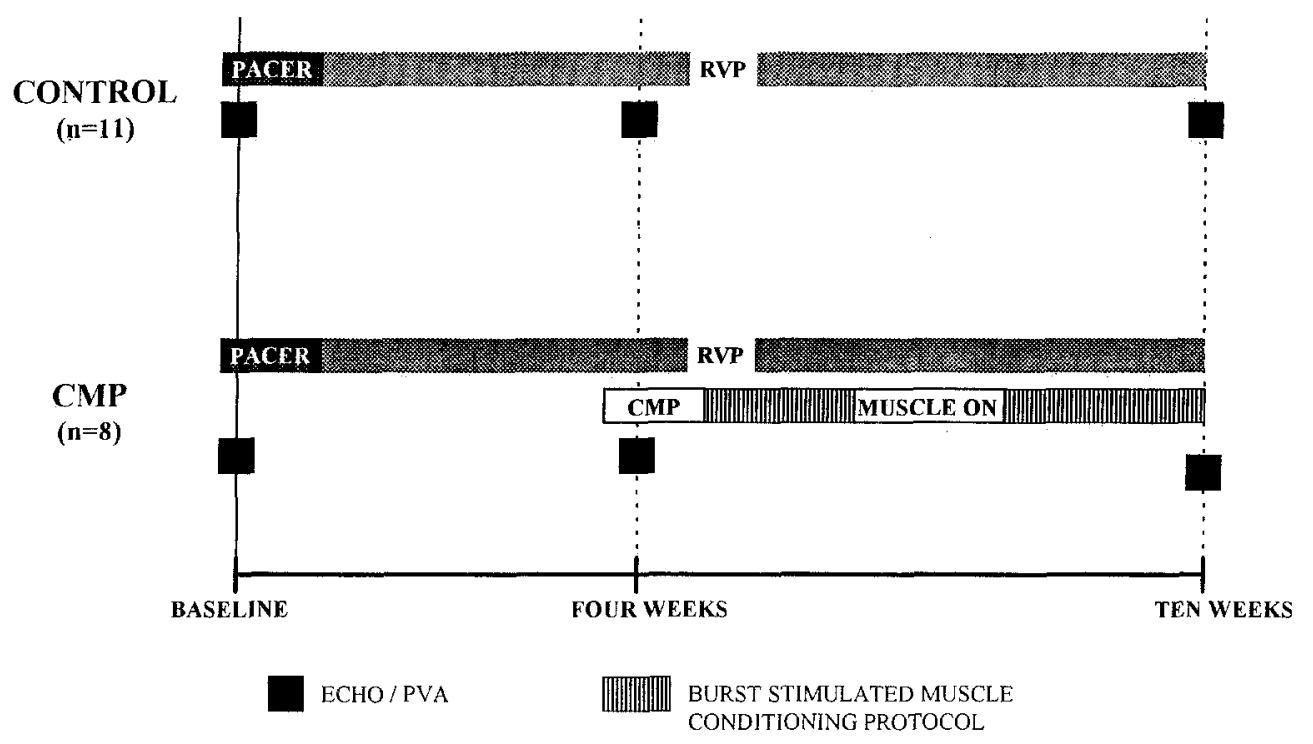

Fig. 1. Experimental design. Our experimental protocol shows that 19 dogs underwent baseline pacemaker installation followed by rapid ventricular pacing $(R V P)$ for 10 weeks at a rate of 215 beats/min to create heart failure. All dogs had pressure-volume analysis $(P V A)$ and two-dimensional echocardiography (echo) studies at baseline and at 4 and 10 weeks of RVP. Eight dogs underwent cardiomyoplasty $(C M P)$ at 4 weeks of RVP. The muscle conditioning protocol is a graded burst-stimulation protocol and is detailed in the text.

in skeletal muscle with continuous electrical stimulation. ${ }^{4,5,18,19}$ In addition, the function of normal hearts is hard to improve. Studies have also frequently relied on the use of load-sensitive indices of function, which may not be able to discern relatively small differences in function. Finally, a number of studies have relied on traditional imaging methods that do not follow the translational motion out of the plane of imaging that occurs with dynamic cardiomyoplasty. ${ }^{20}$

Our study was designed to assess the chronic effects on left ventricular function of a synchronized burst-stimulated conditioned muscle wrap in a model of chronic dilated cardiomyopathy. ${ }^{21}$ In addition, we attempted to determine whether acute dynamic assistance was evident in the setting of heart failure using a tranșformed muscle wrap. We used the techniques of pressure-volume analysis and two-dimensional echocardiography to obtain loadsensitive and load-independent measures of myocardial function. These modalities also allowed us to assess long-term changes in left ventricular volumes.

\section{Method}

All dogs used in this study received care in compliance with the "Guide for Care and Use of Laboratory Animals" published by the National Institutes of Health (NIH publication 86-23, revised 1985), and the investigation was approved by the Institutional Animal Care and Use
Committee of the University of Pennsylvania School of Medicine.

Design of the study. Nineteen heartworm-free adult male mongrel dogs ( 25 to $30 \mathrm{~kg}$ ) were used in this study. Two additional control dogs and three additional cardiomyoplasty-treated dogs died during the course of rapid ventricular pacing and were thus not included in the final analysis. Most of the deaths occurred during the initial development of the model. The protocol is shown schematically in Fig. 1. All dogs underwent baseline two-dimensional echocardiography and pressure-volume analysis as described below. After implantation of pacemakers, all dogs were subjected to rapid ventricular pacing at a rate of 215 beats/min for 4 weeks to ensure development of congestive heart failure.

Studies were repeated to ensure development of cardiac dysfunction before randomization of eight dogs into the cardiomyoplasty group. All 19 dogs continued to undergo the rapid ventricular pacing protocol at 215 beats/min (uninterrupted) for an additional 6 weeks. The eight dogs that had cardiomyoplasty also underwent a concurrent conditioning protocol of graded burst stimulation of the latissimus dorsi muscle wrap during the ensuing weeks of rapid ventricular pacing. Final studies were performed at the end of the 10 -week period. Medical therapy for all dogs consisted of daily digoxin $(0.125 \mathrm{mg})$ and furosemide $(20 \mathrm{mg})$, along with thrice weekly aspirin $(81 \mathrm{mg})$ for the last 9 weeks of rapid ventricular pacing.

Anesthesia. One hour before the operation, each animal was premedicated intramuscularly with acepromazine $(0.1 \mathrm{mg} / \mathrm{kg})$ and glycopyrrolate $(0.001 \mathrm{mg} / \mathrm{kg})$. General anesthesia was induced with ketamine $(10 \mathrm{mg} / \mathrm{kg})$ and diazepam $(0.5 \mathrm{mg} / \mathrm{kg})$ intravenously and maintained after 
Table I. Pressure-volume analysis: Baseline versus 4 weeks of pacing

\begin{tabular}{|c|c|c|c|c|c|c|c|c|}
\hline \multirow[b]{2}{*}{ Parameter } & \multicolumn{3}{|c|}{ Baseline } & \multicolumn{3}{|c|}{ Four weeks of RVP } & \multirow[b]{2}{*}{$\Delta$ Controlf } & \multirow[b]{2}{*}{$\triangle C M P \dagger$} \\
\hline & Control & $C M P$ & $p$ Value* & Control & $C M P$ & $p$ Value* & & \\
\hline Ees $(\mathrm{mm} \mathrm{Hg} / \mathrm{ml})$ & $3.14 \pm 0.70$ & $2.96 \pm 1.51$ & 0.72 & $1.68 \pm 0.89$ & $1.38 \pm 0.23$ & 0.4 & $-46.5 \% \div$ & $-53.4 \%$ \\
\hline PRSW (mm Hg) & $49.9 \pm 3.4$ & $58.7 \pm 7.5$ & 0.003 & $34.6 \pm 9.9$ & $29.2 \pm 8.8$ & 0.27 & $-30.7 \%$ & $-50.3 \% \ddagger$ \\
\hline $\mathrm{dP} / \mathrm{dt} \max (\mathrm{mm} \mathrm{Hg} / \mathrm{sec})$ & $1797 \pm 295$ & $2133 \pm 429$ & 0.06 & $1280 \pm 686$ & $952 \pm 191$ & 0.21 & $-28.8 \% \ddagger$ & $-55.4 \%+$ \\
\hline Peak LVP (mm Hg) & $94.7 \pm 9.5$ & $100.2 \pm 10.4$ & 0.24 & $87.1 \pm 14.3$ & $74.0 \pm 10.8$ & 0.05 & $-8.0 \%$ & $-26.1 \%+$ \\
\hline Heart rate (beats/min) & $121.6 \pm 24.0$ & $131.8 \pm 25.2$ & 0.38 & $123.8 \pm 18.0$ & $112.6 \pm 21.2$ & 0.26 & $+0.02 \%$ & $-14.6 \%$ \\
\hline Tau (msec) & $30.3 \pm 7.2$ & $28.0 \pm 7.9$ & 0.53 & $46.6 \pm 13.7$ & $49.6 \pm 13.8$ & 0.66 & $+53.8 \% \ddagger$ & $+77.1 \%$ \\
\hline $\mathrm{dP} / \mathrm{dt} \min (\mathrm{mm} \mathrm{Hg} / \mathrm{sec})$ & $-1810 \pm 386$ & $-2278 \pm 390$ & 0.02 & $-1437 \pm 581$ & $-1105 \pm 264$ & 0.16 & $+20.6 \% \div$ & $+51.5 \%$ \\
\hline LVEDP $(\mathrm{mm} \mathrm{Hg})$ & $5.2 \pm 1.9$ & $4.9 \pm 2.4$ & 0.24 & $10.7 \pm 5.6$ & $6.0 \pm 2.7$ & 0.05 & $+105.7 \% \div$ & $+22.4 \%$ \\
\hline
\end{tabular}

RVP, Rapid ventricular pacing; $C M P$, cardiomyoplasty; $E$ es, end-systolic elastance; $P R S W$, the slope of the preload recruitable stroke work line; $d P / d t$ max and $d P / d t$ min, maximum and minimum rates of pressure change; $L V P$, left ventricular pressure; $L V E D P$, left ventricular end-diastolic pressure; $T a u$, the time constant of isovolumic pressure decay. All data are expressed as mean \pm standard deviation. For the control group, pressure-volume data are available for 9 of 11 dogs at 4 weeks of RVP; for the cardiomyoplasty group, volume data are available at 4 weeks for 7 of 8 dogs.

*p Value by unpaired $t$-test comparing between control and synchronous wrap groups at that time point.

$\dagger$ The delta columns express the percent change of a hemodynamic variable from baseline to 4 weeks of RVP as a fraction of the its baseline value.

$\ddagger p<0.05$ by repeated-measures analysis of variance followed by Bonferroni's multiple comparison procedure comparing baseline data versus 4 weeks' RVP data within each group.

endotracheal intubation with inhaled oxygen $(3 \mathrm{~L} / \mathrm{min})$ and isoflurane (1\% to $2 \%$ ). All dogs also received perioperative antibiotics.

Instrumentation and data collection. Standard twodimensional transthoracic echocardiograms were obtained before each pressure-volume analysis with the animal awake. Short-axis and apical long-axis and fourchamber views were obtained and stored for later analysis. For the pressure-volume analysis, all dogs were placed under general anesthesia and instrumented under sterile conditions as follows. A $7 \mathrm{~F}$ multielectrode dual-field conductance catheter (Sentron Europe, Maastricht, The Netherlands) and a $5 \mathrm{~F}$ micromanometer-tipped catheter (Millar Instruments, Inc., Houston, Tex.) were placed under fluoroscopic guidance in the left ventricle via cutdowns on the right carotid and right femoral arteries. Similarly, $20 \mathrm{ml}$ occlusion catheters (Applied Vascular, Laguna Hills, Calif.) were placed at the junction of the superior and inferior venae cavae with the right atrium via the right jugular and right femoral veins. A balloon-tipped pulmonary artery catheter was placed via the left jugular vein. Volume measurements were obtained by means of the conductance catheter technique. ${ }^{22-24}$ All hemodynamic signals and the electrocardiogram tracing were processed, digitized at $500 \mathrm{~Hz}$, and stored on computer disk for later analysis.

All data were collected with the ventilator held at end-expiration. For determination of the end-systolic and end-diastolic pressure volume relationships (ESPVR and EDPVR, respectively) and the preload recruitable stroke work relationship (PRSW), the $20 \mathrm{ml}$ balloons in the venae cavae were temporarily inflated to diminish preload, as described previously. ${ }^{23,25-27}$ Each preload reduction and steady-state data collection was performed a minimum of three times with at least 1 minute of recovery time between each sample collection. All incisions were then closed primarily.

Pacemaker insertion. After a recovery period of no less than 3 days after the baseline pressure-volume analysis, each dog underwent placement of modified ventricular pacemakers (model 8341, Medtronics, Inc., Minneapolis, Minn.) designed to maintain prolonged pacing rates at 215 beats $/$ min. Under general anesthesia, a $2 \times 3 \mathrm{~cm}$ section of the apical pericardium was excised through a right anterior thoracotomy, and a unipolar pacing lead (model 6917A, Medtronics) was secured to the left ventricular apex. The lead was then tunneled subcutaneously to a subfascial abdominal pocket and connected to the pacemaker. All incisions were then closed and the pneumothorax was evacuated before extubation. Animals were allowed to recover for at least 7 days, after which pacing was initiated at a rate of 215 beats/min for 4 weeks.

Cardiomyoplasty with the left latissimus dorsi muscle. After 4 weeks of rapid ventricular pacing, pressurevolume analysis and echocardiographic studies were repeated as described earlier. Two control animals did not undergo pressure-volume analysis at 4 weeks but did undergo two-dimensional echocardiography. In addition, one dog ultimately randomized into the cardiomyoplasty group underwent echocardiography and catheterization for pressure measurements alone at 4 weeks of rapid ventricular pacing. Eight of the dogs were randomly selected to undergo cardiomyoplasty immediately after the 4-week rapid ventricular pacing catheterization. Through a left flank incision, the entire latissimus dorsi muscle was mobilized into a pedicle flap based entirely on the thoracodorsal neurovascular bundle. A neuromuscular epimysial electrode (model NMS, Medtronics) was loosely secured over the thoracodorsal nerve just proximal to the trifurcation along the costal surface of the muscle. The mobilized muscle with its lead was then passed into the left hemithorax via a $5 \mathrm{~cm}$ window in the second rib and its humeral insertion was anchored to the periosteum of the first rib. A left thoracotomy through the fifth intercostal space was performed. The muscle was then wrapped posteriorly to anteriorly around both ventricles in a clockwise fashion (as viewed from the apex) and anchored to the surrounding pericardium and epicardium with interrupted pledget-supported monofilament sutures. Adequate care was taken to avoid overstretching 

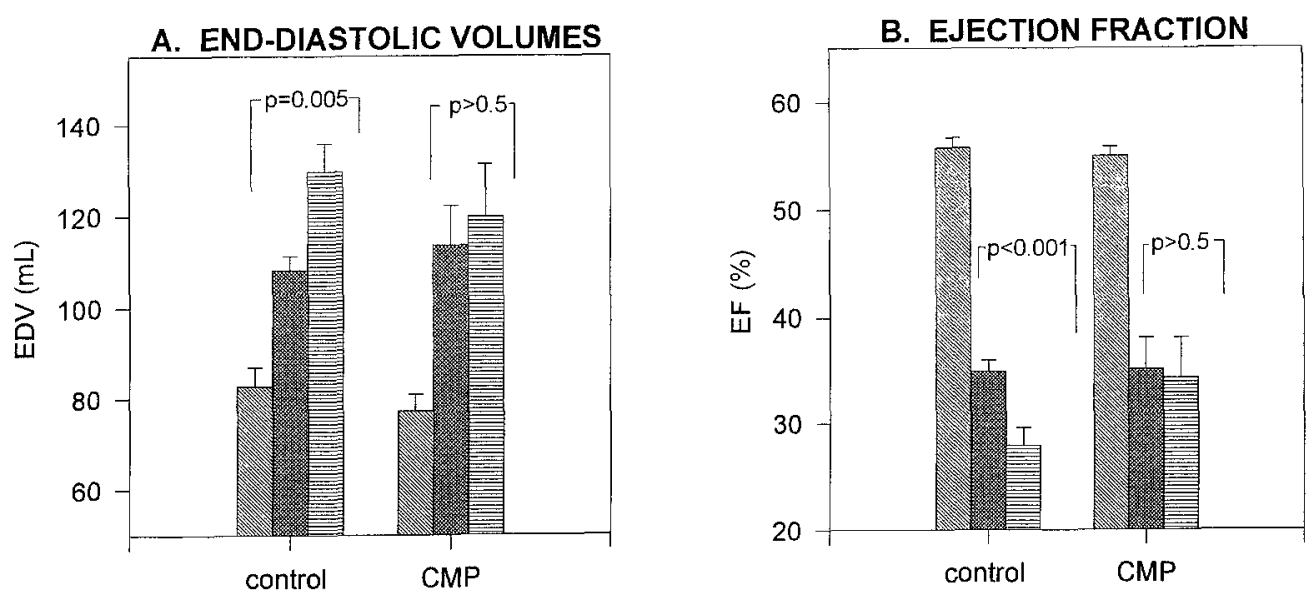

BASELINE

4 WEEKS RVP 10 WEEKS RVP

Fig. 2. Echocardiographic results. These echocardiographic data demonstrate the chronic effects of cardiomyoplasty on ventricular function and geometry. Notice that the initial 4 weeks of rapid ventricular pacing $(R V P)$ increased volumes $(E D V)$ and decreased ejection fraction $(E F)$ in all dogs. In contrast to control dogs, in which this process continued for the last 6 weeks of rapid ventricular pacing, volumes and ejection fractions were stabilized in the cardiomyoplasty $(C M P)$ group. Data represented for the cardiomyoplasty group are with the myostimulator "off" to assess chronic effects of cardiomyoplasty on the failing heart. All $p$ values represent comparisons between 4 and 10 weeks of rapid ventricular pacing within each group.

the muscle or tension on the neurovascular pedicle. The ventral border of the muscle was then anchored to the right ventricular epicardium with a running stitch. The lead was tunneled subcutaneously to a subfascial abdominal pocket and connected to the implantable cardiomyostimulator (model Transform). Once muscle threshold amplitudes were recorded and muscle stimulation was confirmed visually, the stimulator was turned off. A left thoracostomy tube and two subcutaneous drains were placed and all incisions were closed. The tube was removed after at least 1 hour of suction and before recovery from anesthesia.

After a recovery period of no more than 3 days, rapid ventricular pacing was resumed in all dogs, and a concurrent, graded, burst-stimulation muscle conditioning protocol delivered in synchrony with cardiac systole was begun in the cardiomyoplasty group. ${ }^{4}$ The cardiomyostimulator muscle settings were programmed to the following: unipolar mode, pulse width of $198 \mu \mathrm{sec}$, pulse interval of $31 \mathrm{msec}$, adaptation off, and the number of pulses per train (ppt) and synchronization ratio per the conditioning protocol. The pulse amplitude was initially set at twice threshold. Thereafter, the muscle was checked daily by palpation, and the amplitude was increased as required. The muscle conditioning protocol was as follows: week 1 , synchronization ratio of 1:4, muscle:heartbeat, with 1 ppt; week 2 , ratio of 1:3 with $1 \mathrm{ppt}$; week 3 , ratio of $1: 3$ with 2 ppt; week 4 , ratio of $1: 3$ with 3 ppt; and weeks 5 and 6 , ratio of $1: 3$ with 4 ppt. Final studies were performed after a total of 10 weeks rapid ventricular pacing (i.e., at the end of the conditioning protocol). In all dogs the pacemakers were turned off for collection of hemodynamic data.

Data analysis. All hemodynamic data were analyzed off-line with custom software. The conductance catheter was calibrated by the saline method previously described ${ }^{22}$ with the volume gain set to unity. Steady-state parameters that were obtained included heart rate, stroke volume, cardiac output, stroke work, peak left ventricular pressure and end-diastolic left ventricular pressure (LVEDP), maximum and minimum rates of pressure change (maximum $\mathrm{dP} / \mathrm{dt}$ and minimum $\mathrm{dP} / \mathrm{dt}$ ), pulmonary artery pressures, and the time constant of isovolumic pressure decay (tau). Tau was calculated as previously described. ${ }^{28}$ The ESPVR, the EDPVR, and the PRSW data were derived from the preload reduction data. All data in which the heart rate changed more than 5\% were discarded to minimize the effects of cardiovascular reflexes. During collection of myostimulator "on" data, the adaptation was off, and the synchronization delay set so that the myostimulator delivered a $4 \mathrm{ppt}$ burst stimulus just after closure of the mitral valve. The composite ESPVRs and EDPVRs were obtained as detailed in the appendix. Left ventricular volumes according to two-dimensional echocardiography were calculated by the modified biplane Simpson rule. ${ }^{29}$ 

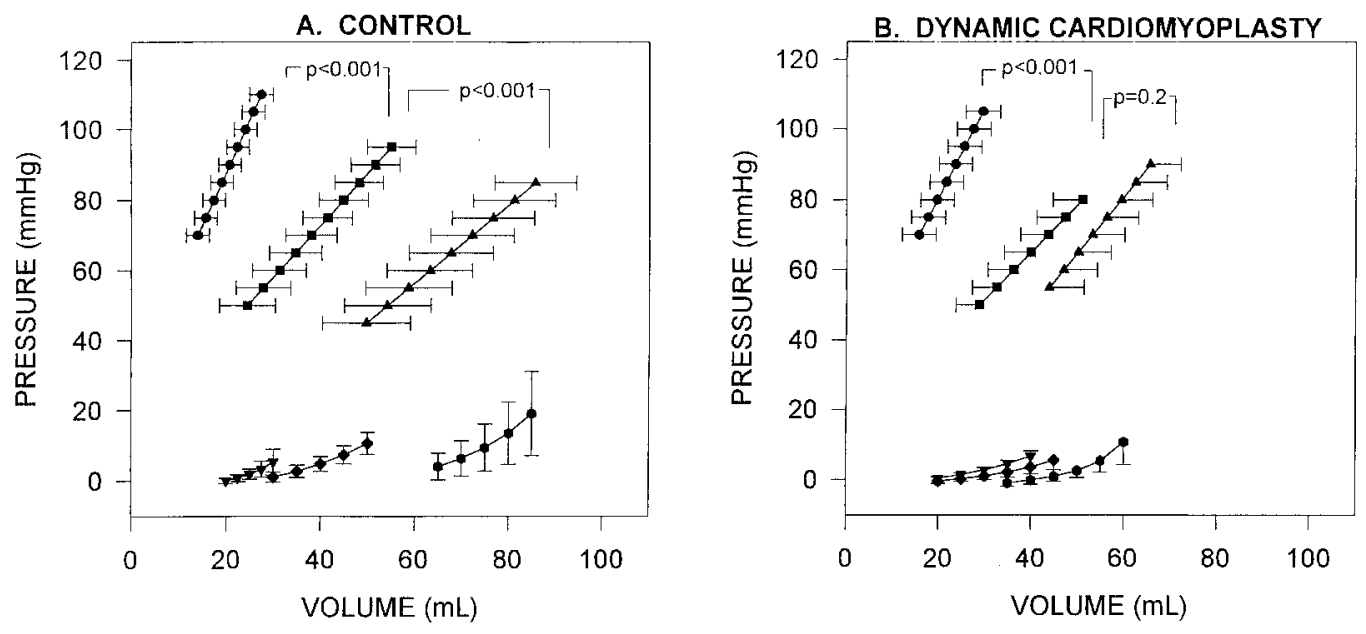

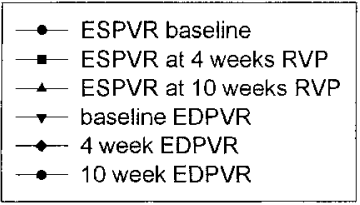

Fig. 3. Effects of rapid ventricular pacing $(R V P)$ and cardiomyoplasty on composite pressure-volume relationships over time. A, The continued rightward shift in both end-systolic (ESPVR) and end-diastolic pressure-volume relations (EDPVR) over the 10-week RVP period in control dogs. This demonstrates that RVP does lead to long-term remodeling throughout the rapid pacing period in our control group. In contrast, in the dynamic cardiomyoplasty group (B), there is an initial rightward shift over the first 4 weeks of RVP only. Dynamic cardiomyoplasty stabilizes the remodeling process during the ensuing 6 weeks despite ongoing rapid pacing. The ESPVR and EDPVR shown in B represent curves obtained with the stimulator "off." The $p$ values reflect the differences in position of the ESPVR between different time points, as indicated by the end-systolic volume for an end-systolic pressure of $80 \mathrm{~mm} \mathrm{Hg}$.

Statistical data were obtained with the use of standard software (SigmaStat, Jandel Scientific, San Rafael, Calif., and SPSS, SPSS Inc., Chicago, III). All data are expressed as mean \pm standard deviation. An unpaired $t$ test was used to determine differences between control and cardiomyoplasty groups. Analysis of variance with repeated measures followed by the multiple comparison method of Bonferroni was used to detect differences between time points within each group. Finally, analysis of variance with repeated measures followed by the multiple comparison method of Dunnett was used to ascertain differences between data obtained with the various stimulator assist modes versus data obtained with the stimulator off.

\section{Results}

Pressure-volume data analysis for all dogs studied at baseline are shown in Table I. All measures of cardiac function except PRSW and maximum negative rate of pressure change (minimum $\mathrm{dP} / \mathrm{dt}$ ) were similar. Volumes and ejection fractions by echocardiography displayed no significant differences at baseline (Fig. 2).

In the time-varying elastance model of the heart, cardiac pressure and volume traverse the pressurevolume plane within the limits of a specific ESPVR (representing systolic performance) and EDPVR (representing passive chamber properties). ${ }^{25} \mathrm{We}$ generated composite ESPVRs and EDPVRs for each group at each time point (Fig. 3, $A$ and $B$, and appendix). These curves allowed us to assess the changes in myocardial performance brought on both by rapid ventricular pacing and by cardiomyoplasty. The curves generated from baseline data (Fig. 3) showed a similar functional state.

By 4 weeks of rapid ventricular pacing, left ventricular function deteriorated according to both load-independent and load-sensitive measures of myocardial performance in both groups (Table I). A comparison between groups showed that functional differences at baseline (PRSW and minimum $\mathrm{dP} / \mathrm{dt}$ ) were not present by 4 weeks of rapid ventricular pacing. Progressive pump failure and left ventricular remodeling was also seen in the composite ESPVRs for both groups (Fig. 3). LVEDP was not signifi- 
Table II. Pressure-volume analysis: Four weeks versus 10 weeks of pacing

\begin{tabular}{|c|c|c|c|c|c|c|c|}
\hline \multirow[b]{2}{*}{ Parameter } & \multicolumn{2}{|c|}{ Four weeks of RVP } & \multicolumn{3}{|c|}{ Ten weeks of RVP } & \multirow[b]{2}{*}{$\Delta$ Controlt } & \multirow[b]{2}{*}{$\Delta C M P+$} \\
\hline & Control & $C M P$ & Control & CMP stim off & $p$ Value $^{*}$ & & \\
\hline Ees $(\mathrm{mm} \mathrm{Hg} / \mathrm{ml})$ & $1.68 \pm 0.89$ & $1.38 \pm 0.23$ & $1.17 \pm 0.32$ & $1.80 \pm 0.53$ & 0.005 & $-30.4 \% \S$ & $+30.4 \%$ \\
\hline PRSW (mm Hg) & $34.6 \pm 9.9$ & $29.2 \pm 8.8$ & $26.3 \pm 4.5$ & $31.3 \pm 5.9$ & 0.05 & $-24.0 \%$ & $+7.2 \%$ \\
\hline $\mathrm{dP} / \mathrm{dt} \max (\mathrm{mm} \mathrm{Hg} / \mathrm{sec})$ & $1280 \pm 686$ & $952 \pm 191$ & $873 \pm 192$ & $985 \pm 230$ & 0.26 & $-31.8 \% \ddagger$ & $+3.5 \%$ \\
\hline Peak LVP (mm Hg) & $87.1 \pm 14.3$ & $74.0 \pm 10.8$ & $79.0 \pm 9.4$ & $84.1 \pm 11.2$ & 0.30 & $-9.3 \%$ & $+13.6 \%$ \\
\hline Heart rate (beats/min) & $123.8 \pm 18.0$ & $112.6 \pm 21.2$ & $115.2 \pm 12.4$ & $115.2 \pm 20.2$ & 0.71 & $-6.9 \%$ & $+2.3 \%$ \\
\hline Tau (msec) & $46.6 \pm 13.7$ & $49.6 \pm 13.8$ & $62.4 \pm 9.0$ & $66.6 \pm 26.5$ & 0.63 & $+33.9 \%$ & $+34.3 \%$ \\
\hline $\mathrm{dP} / \mathrm{dt} \min (\mathrm{mm} \mathrm{Hg} / \mathrm{sec})$ & $-1437 \pm 581$ & $-1105 \pm 264$ & $-1007 \pm 227$ & $-1123 \pm 310$ & 0.36 & $+29.9 \%$ & $+1.6 \%$ \\
\hline LVEDP (mm Hg) & $10.7 \pm 5.6$ & $6.0 \pm 2.7$ & $17.0 \pm 5.1$ & $11.2 \pm 8.2$ & 0.07 & $+58.9 \%$ & $+86.7 \%$ \\
\hline
\end{tabular}

$R V P$, Rapid ventricular pacing; $C M P$, cardiomyoplasty; stim, stimulator; Ees, end-systolic elastance; $P R S W$, the slope of the preload recruitable stroke work line; $d P / d t$ max and $d P / d t$ min, maximum and minimum rates of pressure change; $L V P$, left ventricular pressure; $L V E D P$, left ventricular end-diastolic pressure; Tau, the time constant of isovolumic pressure decay. All data are expressed as mean \pm standard deviation. For the control group, pressure-volume data are available for 9 of 11 dogs at 4 weeks of RVP. For the synchronous wrap group, volume data are available for 7 of 8 dogs at 4 weeks.

* $\mathrm{p}$ Value by unpaired $t$-test comparing between control and synchronous wrap groups at ten weeks.

$\dagger$ The delta columns express the percent change of a hemodynamic variable from four weeks to ten weeks RVP as a fraction of the its 4 week RVP value. $\ddagger p<0.05$ by repeated-measures analysis of variance followed by Bonferroni's multiple comparison procedure comparing 4 weeks' RVP versus 10 weeks' RVP within each group.

$\S p<0.1$ by repeated-measures analysis of variance followed by Bonferroni's multiple comparison procedure comparing 4 weeks' RVP versus 10 weeks' RVP within each group.

Table III. Pressure-volume analysis: Dynamic effects at ten weeks of pacing

\begin{tabular}{|c|c|c|c|c|c|}
\hline \multirow[b]{2}{*}{ Parameter } & \multicolumn{5}{|c|}{ Ten weeks of $R V P$} \\
\hline & $\begin{array}{l}\text { Stimulator } \\
\text { off }\end{array}$ & $\begin{array}{l}\text { Augmented } \\
\text { stim } 1: 2 \text { beats }\end{array}$ & $\begin{array}{l}\text { Nonaugmented } \\
\text { stim } 1: 2 \text { beats }\end{array}$ & Stimulator 1:1 & $\begin{array}{l}\text { ANOVA } \\
\text { p value* }\end{array}$ \\
\hline Ees $(\mathrm{mm} \mathrm{Hg} / \mathrm{ml})$ & $1.80 \pm 0.53$ & $2.19 \pm 0.69$ & $1.97 \pm 0.68$ & $2.49 \pm 0.58^{*}$ & 0.009 \\
\hline PRSW (mm Hg) & $31.3 \pm 5.9$ & $40.6 \pm 10.8^{*}$ & $34.5 \pm 12.1$ & $45.4 \pm 11.0^{*}$ & 0.004 \\
\hline $\mathrm{dP} / \mathrm{dt} \max (\mathrm{mm} \mathrm{Hg} / \mathrm{sec})$ & $985 \pm 230$ & $1003 \pm 266$ & $934 \pm 253$ & $1034 \pm 234$ & 0.40 \\
\hline Stroke work $(\mathrm{mm} \mathrm{Hg} \cdot \mathrm{ml})$ & $1416 \pm 539$ & $1707 \pm 973$ & $1308 \pm 561$ & $1648 \pm 724$ & 0.06 \\
\hline Peak LVP (mm Hg) & $84.1 \pm 11.2$ & $85.2 \pm 10.4$ & $81.0 \pm 10.1$ & $84.6 \pm 6.5$ & 0.55 \\
\hline Heart rate (beats/min) & $115.2 \pm 20.2$ & $115.6 \pm 17.9$ & $115.3 \pm 19.4$ & $116.2 \pm 18.6$ & 0.98 \\
\hline Tau (msec) & $66.6 \pm 26.5$ & $67.4 \pm 29.3$ & $61.1 \pm 21.7$ & $59.0 \pm 19.1$ & 0.41 \\
\hline $\mathrm{dP} / \mathrm{dt} \min (\mathrm{mm} \mathrm{Hg} / \mathrm{sec})$ & $-1123 \pm 310$ & $-1168 \pm 311$ & $-1140 \pm 298$ & $-1238 \pm 266$ & 0.45 \\
\hline LVEDP (mm Hg) & $11.2 \pm 8.2$ & $9.0 \pm 4.3$ & $8.6 \pm 4.4$ & $9.8 \pm 5.1$ & 0.11 \\
\hline
\end{tabular}

RVP, Rapid ventricular pacing; Ees, end-systolic elastance; $P R S W$, the slope of the preload recruitable stroke work line; $d P / d t$ max and $d P / d t$ min, maximum and minimum rates of pressure change; $L V P$, left ventricular pressure; $L V E D P$, left ventricular end-diastolic pressure; Tau, the time constant of isovolumic pressure decay. All data are expressed as mean \pm standard deviation. Data for the $1: 2$ beats are analyzed separately for augmented and nonaugmented beats. $N=8$ dynamic CMP dogs

${ }^{*} p<0.05$ by Dunnett's multiple comparison procedue following the repeated-measures analysis of variance comparing stimulator on settings versus stimulator "off."

cantly elevated by 4 weeks of rapid ventricular pacing in the synchronous wrap group (furosemide withheld only morning of 4-week study/cardiomyoplasty in the cardiomyoplasty group, but withheld morning before and morning of 4-week study in controls). However, the load-independent EDPVR shows similar diastolic creep (i.e., rightward shift of the EDPVR) in both groups. Echocardiographic data (see Fig. 2) also showed a significant increase of volumes and a significant decrease of ejection fraction by 4 weeks of rapid ventricular pacing in both groups. No statistically significant difference could be detected between groups.
Two of the 11 dogs in the control group had no pressure-volume analysis data at the 4-week time point. However, echocardiographic data from those two dogs demonstrated end-diastolic volumes and ejection fractions within the range seen for the group. In addition, the single dog in the cardiomyoplasty group that had no volume data at the 4-week time point had pressure and imaging data that were within the range seen for the group.

By the end of the 10-week pacing period, pump performance continued to deteriorate in control animals. All parameters of systolic function were lower (end-systolic elastance, $p=0.1$; PRSW, $p=$ 
0.006; maximum $\mathrm{dP} / \mathrm{dt}, p=0.04$ ), and diastolic performance measures of LVEDP, minimum $\mathrm{dP} / \mathrm{dt}$, and tau were also significantly different $(p<0.05)$ from their 4-week time points (Table II). Echocardiographic left ventricular volumes continued to show progressive enlargement $(p=0.005)$ and deterioration in ejection fraction $(p<0.001$, Fig. 2). Finally, the composite ESPVR and EDPVR of control dogs also confirmed deteriorating pump performance and diastolic creep (see Fig. 3, $A$ ).

In contrast, the results from the cardiomyoplasty group show chronic stabilization of pump function. All data for assessment of chronic effects of cardiomyoplasty were performed with the myostimulator off. In addition, end-systolic elastance improved by 10 weeks of rapid ventricular pacing and was significantly different from control values at that time $(p=0.005)$. PRSW also stabilized in the wrap group, and the difference from control values at 10 weeks was also significant $(p=0.05)$. Load-sensitive measures of contractility (maximum $\mathrm{dP} / \mathrm{dt}$ and peak left ventricular pressures) stabilized in the cardiomyoplasty group. Diastolic performance showed evidence of stabilization when measured by the loadsensitive parameter of minimum $\mathrm{dP} / \mathrm{dt}$ (see Table II). End-diastolic pressures and tau increased in the cardiomyoplasty group (as a result of different diuretic management as stated earlier). ${ }^{28}$

The final 6 weeks of rapid ventricular pacing also showed a persistent rightward shift in the composite ESPVR, along with continued diastolic creep in control animals (Fig. 3, A). Similarly, an increase in ventricular volumes and a decrease in ejection fraction was obtained by echocardiography (end-diastolic volume, ejection fraction, $p<0.01$, Fig. 2). In contrast, dynamic cardiomyoplasty not only stabilized echocardiographic volume and ejection fraction (end-diastolic volume, ejection fraction, $p>0.5$, Fig. 2) but also maintained the composite ESPVR and EDPVR curves at their 4-week positions (Fig. 3, B).

Pressure-volume data were also obtained with the myostimulator at the 1:2 muscle/heartbeat ratio and 1:1 settings to assess acute dynamic effects of cardiomyoplasty (Table III). Load-independent indices of end-systolic elastance and PRSW for the 1:1 beats demonstrate dynamic assistance $(p<0.05)$, and similar results are seen for the augmented 1:2 beats (PRSW, $p<0.05$ ). Stroke work was also marginally greater for these beats. Although no significant difference was noted in diastolic indices (minimum $\mathrm{dP} / \mathrm{dt}$ and LVEDP), nonetheless all values for these assisted beats improved. The nonaugmented 1:2

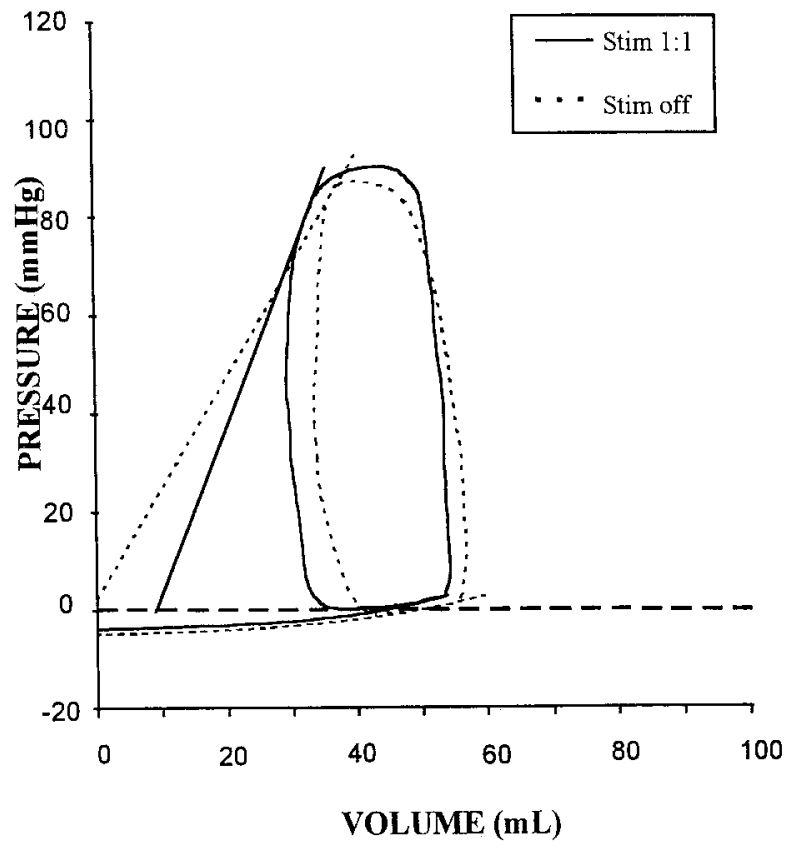

Fig. 4. Effect of dynamic assistance on the pressurevolume relationships in heart failure. Typical results are depicted for one dog during dynamic assistance for every beat (stimulator 1:1, solid lines) in comparison with unassisted beats (stimulator "off," dotted lines). Stroke work and the EDPVR do not change significantly for the assisted beats. Similarly, the position of the ESPVR and the pressure-volume loops do not change. However, a change in the end-systolic elastance (the slope of the ESPVR) for the assisted beats is evident when compared with unassisted beats. Our composite ESPVRs and EDPVRs (not shown) comparing assisted $1: 1$ and $1: 2$ beats versus unassisted beats show similar results. This is an increase in effective contractility with skeletal muscle assistance. Because the stroke work (area within the pressure-volume loop) is not changed with assistance, myocardial workload is therefore decreased despite an increase in effective contractility.

beats showed no difference in function when compared with myostimulator "off" settings. Finally, there was no change in the volume axis position of the composite ESPVRs for the assisted beats when compared with unassisted beats (data not shown). A representative example, from one $\mathrm{dog}$, of the changes in the pressure-volume relations and pressure-volume loops brought on by skeletal muscle assistance, is shown in Fig. 4.

\section{Discussion}

Dynamic cardiomyoplasty is a poorly understood surgical treatment for end-stage heart failure. Pa- 


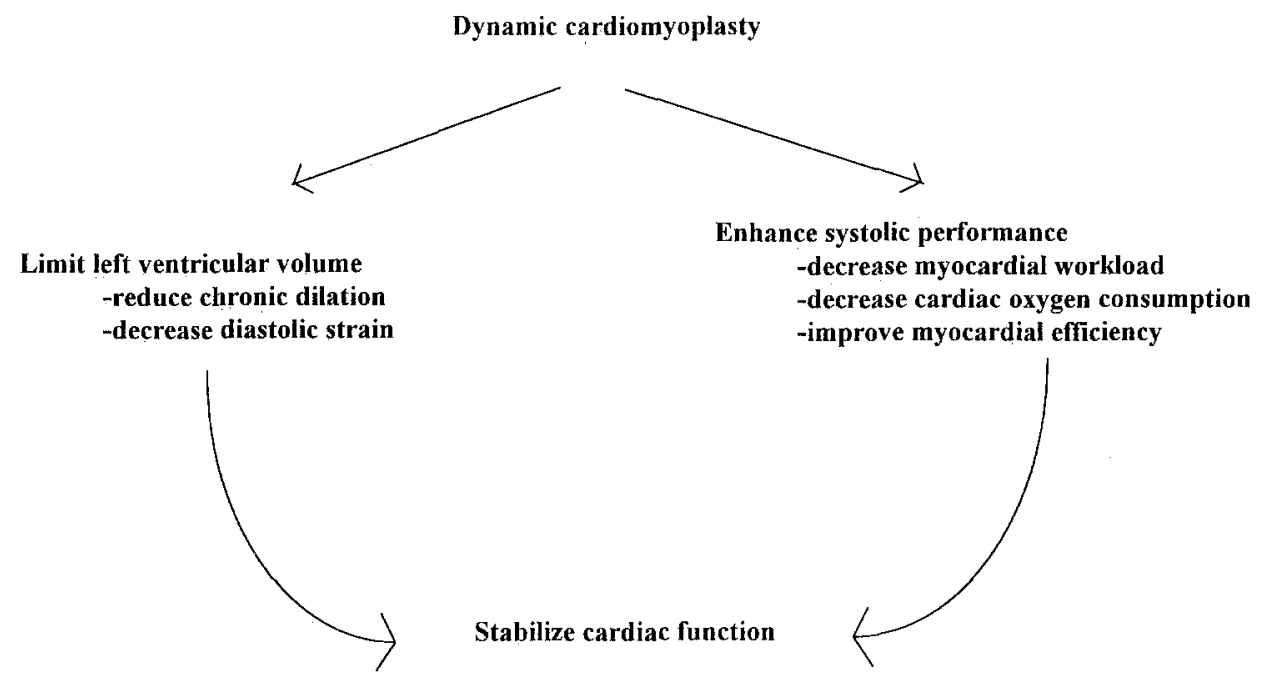

Fig. 5. Proposed mechanisms of cardiomyoplasty. Dynamic cardiomyoplasty acts by at least two mechanisms. Girdling effects on the left ventricle limit the chronic dilation associated with heart failure and thus decrease diastolic strain. Enhancement of effective contractility with skeletal muscle assistance decreases myocardial workload and thus decreases cardiac oxygen consumption and increases myocardial efficiency. Both of these effects presumably act to stabilize cardiac function over time.

tients receiving this treatment typically exhibit improvement in symptoms, but consistent objective improvement in traditional indices of cardiac function in these same patients is sometimes lacking. ${ }^{1-17}$ Postulated mechanisms of action include both acute systolic assistance ${ }^{6-14}$ and a passive girdling effect on the left ventricle. ${ }^{3,15-17} \mathrm{We}$ undertook this study to assess both effects on the left ventricle in a clinically relevant fashion, that is, with the use of a synchronized burst-stimulated conditioned muscle wrap in a model of chronic heart failure.

Rapid ventricular pacing of dog hearts has been previously validated as a model of severe biventricular dilated cardiomyopathy, morphologically, neurohormonally, and functionally. ${ }^{21}$ Our model is a modification of the existing well-characterized model. Most reports use rapid ventricular pacing at levels of 240 to 260 beats/min to create a dilated cardiomyopathy within 2 to 4 weeks. When pacing is continued for protracted periods at this level, the mortality rate is significant. Our modification was a decrease in the pacing rate to 215 beats/min to create this model by 4 weeks of rapid ventricular pacing. This change, however, also allowed us to maintain the rapid pacing for 10 weeks so that conditioning of the muscle wrap and the final study could occur in the setting of heart failure. Assessment of cardiac function in our study included traditional imaging methods (echocardiography), along with pressure-volume analysis to obtain loadindependent assessments of function.

Our results show that in the setting of chronic severe heart failure, cardiomyoplasty affects cardiac function by at least two mechanisms (Fig. 5). Longterm effects of cardiomyoplasty include an attenuation of the ventricular enlargement and the remodeling process that results in heart failure. Girdling effects have been demonstrated in several other recent studies. ${ }^{3,15-17}$ Remodeling was demonstrated in our study by a rightward shift in composite ESPVR and EDPVR in control hearts throughout the rapid pacing period. This is an expected finding, because progressive left ventricular dilation is a common feature of severe congestive heart failure and is accompanied by rightward shifts of both the ESPVR and the EDPVR. Girdling effects in our study are demonstrated by a lack of rightward shifts in the pressure-volume relations after cardiomyoplasty, despite ongoing rapid pacing. Chronic overloading of the heart, as seen in dilated cardiomyopathy, leads to progression of heart failure and remodeling. ${ }^{30}$ This is associated with alterations of the extracellular matrix, myocyte cytoskeletal arrangements, myocyte alignment, and contractile protein orientation, all of which change myocyte geometry and function. ${ }^{21,30}$ It is possible that the presence of additional limiting structures around the heart with a low elastance, such as the condi- 
tioned muscle wrap, has decreased these processes to stop the progressive dilation seen in heart failure.

Chronic effects may also be partly due to the phenotypic changes that occur during electrical transformation of the skeletal muscle wrap. ${ }^{4,5,18,19}$ Transformation of skeletal muscle has been shown to decrease muscle mass, area, and fiber caliber. This wrap may act like a volume-stabilizing girdle, because it shrinks around the heart during the process of transformation from type II to type I fibers. This shrinkage, which has been considered an undesirable side effect of muscle transformation, may play a larger role in the improvement seen after cardiomyoplasty than previously thought.

Cardiomyoplasty also appears to act dynamically to increase the apparent contractile state of the heart (see Figs. 4 and 5). Skeletal muscle assistance was shown in Jatene's series ${ }^{6}$ of 13 patients. Cho, ${ }^{8}$ Aklog, ${ }^{9}$ and their colleagues have shown augmentation of elastance for assisted beats in normal hearts. Other reports have shown improvement in hemodynamics with synchronized stimulation optimization. ${ }^{12}$ In addition, several recent studies have demonstrated decreased wall stress with added systolic compression. ${ }^{10,11}$ In our study on cardiomyopathic hearts, both stimulator $1: 2$ assisted and $1: 1$ beats showed an increase in load-independent indices of contractility (end-systolic elastance and PRSW) when compared with stimulator "off" results. Composite ESPVRs and EDPVRs do not show a shift along the volume axis with dynamic assistance. Data from a typical dog (see Fig. 4) show an increase in the slope of the ESPVR, with minimal change in the EDPVR, the pressure-volume loop, or the position along the volume axis of the ESPVR with skeletal muscle stimulation. This implies a decrease in the myocardial workload, because the added effective contractility yielding a similar amount of stroke work is due to the skeletal muscle contraction.

In summary, our study assessed both chronic and dynamic effects of a synchronized stimulated muscle wrap in a model of severe heart failure. We conclude (see Fig. 5) that the presence of a transformed muscle wrap in cardiomyoplasty attenuates both ventricular dilation and the remodeling process associated with severe progressive heart failure. We also believe that cardiomyoplasty acts by an acute dynamic process by increasing apparent contractility and by decreasing myocardial workload.

We gratefully acknowledge the excellent technical assistance of Mr. Randall Rossi and Dr. Y.-G. Liu. We also thank Medtronic, Inc., and in particular Kendra Gealow, $\mathrm{PhD}$, and James Cox, BSE, for providing our laboratory with essential equipment. Finally, we thank Joseph Gorman, MD, and Harvey Kushman, PhD, for their help with the statistical analysis performed in this work.

\section{REFERENCES}

1. Carpentier A, Chachques JC, Acar C, Relland J, Mihaileanu $\mathrm{S}$, Bensasson $\mathrm{K}$, et al. Dynamic cardiomyoplasty at seven years. J Thorac Cardiovasc Surg 1993;106:42-54.

2. Furnary AP, Moreira LFP, Jessup M, and the American Cardiomyoplasty Group. Dynamic cardiomyoplasty improves systolic ventricular function. Circulation 1994;90(Suppl): I309.

3. Hagege AA, Desnos M, Fernandez F, Besse B, Mirochnik N, Castaldo $M$, et al. Clinical study of the effects of latissimus dorsi muscle flap stimulation after cardiomyoplasty. Circulation 1995:92(Suppl):II210-5.

4. Lee KF, Wechisler AS. Dynamic cardiomyoplasty. Adv Card Surg 1993:4:207-36.

5. El Oakley RM, Jarvis JC. Cardiomyoplasty-a critical review of experimental and clinical results. Circulation 1994;90: 2085-90.

6. Jatene AD, Moriera LFP, Stolf NAG, Bocchi EA, Seferian P, Fernandes PMP, et al. Left ventricular function changes after cardiomyoplasty in patients with dilated cardiomyopathy. J Thorac Cardiovasc Surg 1991;102:132-9.

7. Chachques JC, Grandjean PA, Schwartz K, Mihaileanu S, Fardeau M, Swynghedauw B, et al. Effects of latissimus dorsi dynamic cardiomyoplasty on ventricular function. Circulation 1988;78(Suppl):III203-16.

8. Cho PW, Levin HR, Curtis WE, Tsitlik JE, DiNatale JM, Kass DA, et al. Pressure-volume analysis of changes in cardiac function in chronic cardiomyoplasty. Ann Thorac Surg 1993;56:38-45.

9. Aklog L, Murphy MP, Chen FY, Smith WJ, Laurence RG, Appleyard RF, et al. Right latissimus dorsi cardiomyoplasty improves left ventricular function by increasing peak systolic elastance. Circulation 1994;90(Suppl):II112-9.

10. Lee KJ, Dignan RJ, Parmar JM; Dyke CM, Benton G, Yeh T, et al. Effects of dynamic cardiomyoplasty on left ventricular performance and myocardial mechanics in dilated cardiomyopathy. J Thorac Cardiovasc Surg 1991;102:124-31.

11. Chen FY, Aklog L, deGuzman BJ, Laurence RG, Couper GS, Appleyard RF, et al. New technique measures decreased transmural myocardial pressure in cardiomyoplasty. Ann Thorac Surg 1995;60:1678-82.

12. Oh JH, Badhwar V, Chiu RCJ. Mechanisms of dynamic cardiomyoplasty: current concepts. J Card Surg 1996;11: 194-9.

13. Kawaguchi O, Goto Y, Fútaki S, Ohgoshi Y, Yaku H, Suga H. Mechanical enhancement and myocardial oxygen saving by synchronized dynamic left ventricular compression. J Thorac Cardiovase Surg 1992;103:573-81.

14. Millner RWJ, Burrows M, Pearson I, Pepper JR. Dynamic cardiomyoplasty in chronic left ventricular failure: an experimental model. Ann Thorac Surg 1993;55:493-501.

15. Capouya ER, Gerber RS, Drinkwater DC, Pearl IM, Sack JB, Aharon AS, et al. Girdling effect of nonstimulated cardiomyoplasty on left ventricular function. Ann Thorac Surg 1993; 56:867-71.

16. Kass DA, Baughman KL, Pak PH, Cho PW, Levin HR, 
Gardner TJ, et al. Reverse remodeling from cardiomyoplasty in human heart failure. Circulation 1995;91:2314-8.

17. Nakajima H, Niinami H, Hooper TL, Hammond RL, Nakajima $\mathrm{HO}, \mathrm{Lu} \mathrm{L}$, et al. Cardiomyoplasty: probable mechanism of effectiveness using the pressure-volume relationship. Ann Thorac Surg 1994;57:407-15.

18. Salmons S, Sreter FA. Significance of impulse activity in the transformation or skeletal muscle type. Nature 1976;263: 30-4.

19. Mannion ID, Bitto T, Hammond RL, Rubenstein NA, Stephenson LW. Histochemical and fatigue characteristics of conditioned canine latissimus dorsi muscle. Circ Res 1986; 58:298-304.

20. Cho PW, Levin HR, Moore CC, Tsitlik JE, McVeigh ER, Gardner TJ, et al. New method for mechanistic studies of cardiomyoplasty: three dimensional MR reconstructions. Ann Thorac Surg 1994;57:1605-11.

21. Spinale FG. Pathophysiology of tachycardia induced heart failure. New York: Futura; 1996.

22. Baan $\mathbf{J}$, van der Velde ET, de Bruin HG, Smeek GJ, Koops $\mathrm{J}$, van Dijk $\mathrm{AD}$, et al. Continuous measurement of the left ventricular volume in animals and humans by the conductance catheter. Circulation 1984;70:812-23.

23. Kass DA. Clinical evaluation of left heart function by conductance catheter technique. Eur Heart J 1992;13(Suppl E):7-64.

24. Burkhoff D. The conductance method of left ventricular volume estimation-methodologic limitations put into perspective. Circulation 1990;81:703-6.

25. Sagawa K, Maughan L, Suga H, Sunagawa K. Cardiac contraction and the pressure-volume relationship. Oxford: Oxford University Press; 1988.

26. Glower DD, Spratt JA, Snow ND, Kabas JS, Davis JW, Olsen $\mathrm{CO}$, et al. Linearity of the Frank-Starling relationship in the intact heart: the concept of preload recruitable stroke work. Circulation 1985;71:994-1009.

27. Kass DA, Yamazaki T, Burkhoff D, Maughan WL, Sagawa K. Determination of left ventricular end-systolic pressure-volume relationships by the conductance (volume) catheter technique. Circulation 1986;73:586-95.
28. Raff GL, Glantz SA. Volume loading slows left ventricular isovolumic relaxation rate. Circ Res 1981;48:813-24.

29. Schiller NB, Shah PM, Crawford M, DeMaria A, Devereux $\mathrm{R}$, Feigenbaum $\mathrm{H}$, et al. Recommendations for quantitation of the left ventricle by two dimensional echocardiography. J Am Soc Echocardiol 1989;2:358-67.

30. Eichhorn EJ, Bristow MR. Medical therapy can improve the biological properties of the chronically failing heart. Circulation 1996;94:2285-96.

\section{Appendix}

Generation of the composite ESPVRs and EDPVRs. The ESPVRs were obtained by using each dog's individual end-systolic elastance $\left(E_{e s}\right)$ along with its volume intercept $\left(\mathrm{V}_{0}\right)$. End-systolic volumes $\left(\mathrm{V}_{\mathrm{es}}\right)$ for the composite ESPVR were then obtained from a preset range of $5 \mathrm{~mm}$ $\mathrm{Hg}$ increments of end-systolic pressures $\left(\mathrm{P}_{\mathrm{es}}\right)$ using the equation:

$$
\mathrm{V}_{\mathrm{es}}=\left(\mathrm{P}_{\mathrm{es}} / \mathrm{E}_{\mathrm{es}}\right)+\mathrm{V}_{0}
$$

End-systolic volumes were then averaged at each selected pressure for the composite ESPVR. The range of pressures was obtained from the range seen during typical preload reduction.

Similarly, EDPVRs were constructed by determining the end-diastolic pressures $\left(\mathrm{P}_{\mathrm{ed}}\right)$ from $5 \mathrm{ml}$ increments of a range of end-diastolic volumes $\left(\mathrm{V}_{\mathrm{ed}}\right)$. The EDPVR was fit to a monoexponential equation:

$$
\mathbf{P}_{\mathrm{ed}}=\mathrm{P}_{0}+\mathrm{a}\left(e^{\mathrm{bVed}}+1\right)
$$

where $P_{0}$, $a$ and $b$ are the constants obtained from each $\operatorname{dog}$ at each time point, and $\mathrm{e}$ is the base of the natural logarithm. End-diastolic pressures from each volume increment were then averaged to obtain the composite EDPVR. The preset volume range was determined by using those points where pressure was calculated to be within the range seen during preload reduction. 